\title{
Dépendance sans intégration: La cosubordination comme mode de jonction propositionnelle et sa pertinence en acquisition du français $L 2$
}

\author{
Manon Buysse \\ Vrije Universiteit Brussel, Belgique \\ Université de Helsinki, Finlande \\ mbuysse@vub.ac.be
}

\begin{abstract}
Résumé. La jonction propositionnelle, ou le processus visant à combiner plusieurs propositions dans un énoncé complexe, est un phénomène largement décrit en linguistique française (Bronckart \& Schneuwly 1984, Clark 1998, Kern 2000). Dans un contexte d'acquisition du français comme langue seconde (FL2), en particulier, la complexification propositionnelle est un des objets de recherche centraux (Benazzo 2004, Kerr-Barnes 1998, Véronique 2005, Welcomme 2013). Certaines études axées sur le développement global d'apprenants du français exploitent en outre la jonction de propositions comme un indice essentiel pour évaluer la progression dans la maîtrise de la langue seconde (Bartning \& Kirchmeyer 2003, Schlyter 2003, Bartning \& Schlyter 2004). Le cadre d'analyse généralement adopté pour décrire le développement de la jonction propositionnelle est la bipartition traditionnelle entre la coordination d'une part (Georges est malade et il ne veut pas manger) et la subordination d'autre part (Georges dit qu'il est malade, complétive, ou George ne veut pas manger parce qu'il est malade, adverbiale). Pourtant, cette opposition binaire ne rend pas compte de l'écart très important entre le moment d'acquisition d'énoncés comme Quand il pleut, je prends un parapluie et celui de phrases composées comme Je connais l'homme dont tu me parles. De plus, elle ne permet pas la description adéquate de types de jonction propositionnelle tels que Il veut laver la voiture et J'essayerai de venir à ta fête. Pour permettre une approche plus affinée de la jonction propositionnelle, la Grammaire du Rôle et de la Référence (Role and Reference Grammar ou RRG ; Van Valin \& LaPolla 1997, Van Valin 2005) avance un troisième type de relation syntaxique entre propositions, la cosubordination. Nous évaluerons l'apport de la tripartition proposée pour l'acquisition d'une L2 en analysant le développement progressif de la jonction propositionnelle chez des apprenants néerlandophones de FL2. La présente contribution se pose l'objectif de mettre en évidence les insuffisances de la saisie de la jonction propositionnelle au moyen de la bipartition traditionnelle corrdination/subordination. Elle présente l'alternative avancée par la RRG, sur laquelle elle s'appuie pour décrire le processus d'appropriation de la connexion de propositions en FL2 par des apprenants néerlandophones. Elle discute finalement l'apport de la tripartition pour une meilleure appréhension du processus de jonction et sa pertinence pour la recherche en acquisition de langues étrangères.
\end{abstract}

\begin{abstract}
Clause linking, or the combination of multiple predications into one syntactically complex whole, has been widely studied in French linguistics (Bronckart \& Schneuwly 1984, Clark 1998, Kern 2000). In the context of French second language acquisition, in particular, clause complexification is a highly popular research topic (Benazzo 2004, Kerr-Barnes 1998, Véronique 2005, Welcomme 2013) ; to some, it serves as a metric for evaluating learners' progressive mastery of the target language (Bartning \& Kirchmeyer 2003, Schlyter 2003). The analytical framework traditionally adopted for the description of clause linking makes a binary distinction between coordination (George feels sick and he doesn't want to eat) and subordination (George says that he feels sick or George doesn't want to eat because he feels sick). This opposition, however, does not account for obvious variations in terms of moment of acquisition between, for instance, When it rains, I grab an umbrella and such composite sentences like I know the man of whom you're speaking. In addition, it does not allow for an adequate description of such forms of clause linkage as He wants to wash the car and I'll try to come to the party. As an alternative to the traditional dichotomy, the framework of Role and Reference Grammar
\end{abstract}


(RRG; Van Valin \& LaPolla 1997, Van Valin 2005) proposes a third type of syntactic relation between clauses, i.e. cosubordination. The present contribution will discuss the imperfections of the coordination/subordination dichotomy and will evaluate the value of RRG's proposed tripartition for the study of clause linking, especially in second language acquisition, by applying the framework to the oral productions of L2 French learners at different stages of development (Buysse \& De Clercq 2014, Buysse 2014, Buysse 2015).

La jonction propositionnelle, ou le processus visant à combiner plusieurs propositions dans un énoncé complexe, est un phénomène largement décrit en linguistique française (Bronckart \& Schneuwly, 1984 ; Clark, 1998 ; Kern, 2000). Dans un contexte d'acquisition du français comme langue seconde (FL2), en particulier, la complexification propositionnelle est un des objets de recherche centraux (Benazzo, 2004 ; Kerr-Barnes, 1998 ; Véronique, 2005 ; Welcomme, 2013). Certaines études axées sur le développement global d'apprenants du français exploitent en outre la jonction de propositions comme un indice essentiel pour évaluer la progression dans la maîtrise de la langue seconde (Bartning \& Kirchmeyer, 2003 ; Schlyter, 2003 ; Bartning \& Schlyter, 2004).

Le cadre d'analyse généralement adopté pour décrire le développement de la jonction propositionnelle est la bipartition traditionnelle entre la coordination d'une part (Georges est malade et il ne veut pas manger) et la subordination d'autre part (Georges dit qu'il est malade, complétive, ou George ne veut pas manger parce qu'il est malade, adverbiale). Pourtant, cette opposition binaire ne rend pas compte de l'écart très important entre le moment d'acquisition d'énoncés comme Quand il pleut, je prends un parapluie et celui de phrases composées comme Je connais l'homme dont tu me parles. De plus, elle ne permet pas la description adéquate de types de jonction propositionnelle tels que Il veut laver la voiture et J'essayerai de venir à ta fête. Pour permettre une approche plus affinée de la jonction propositionnelle, la Grammaire du Rôle et de la Référence (Role and Reference Grammar ou RRG ; Van Valin \& LaPolla, 1997 ; Van Valin, 2005) avance un troisième type de relation syntaxique entre propositions, la cosubordination. Nous évaluerons l'apport de la tripartition proposée pour l'acquisition d'une L2 en analysant le développement progressif de la jonction propositionnelle chez des apprenants néerlandophones de FL2 (Buysse \& De Clercq, 2014 ; Buysse, 2014 ; Buysse, 2015).

Dans la première section de cette contribution, nous fournirons un aperçu de la recherche existante concernant le développement de la jonction propositionnelle en français langue seconde. Nous mettrons également en évidence les insuffisances de sa saisie au moyen de la bipartition traditionnelle coordination/subordination. La section 2 présentera en détail l'alternative avancée par la RRG. La section 3 exploitera le cadre de la RRG pour décrire le processus d'appropriation de la connexion de propositions en FL2 par des apprenants néerlandophones. La quatrième section, finalement, discutera l'apport de la tripartition pour une meilleure appréhension du processus de jonction et formulera des conclusions au sujet de sa pertinence pour la recherche en acquisition de langues étrangères.

\section{La jonction propositionnelle en français L2}

La plupart des études apparues jusqu'ici sur le développement de la jonction propositionnelle en français langue seconde fondent leurs conclusions sur des données essentiellement orales, notamment des narrations orales, personnelles ou basées sur des supports visuels (comme l'histoire de la grenouille; Mayer, 1969). Elles fournissent des observations convergentes concernant la maîtrise progressive des mécanismes de jonction par les apprenants du français L2.

Les apprenants débutants du français organisent généralement leur discours selon le " principe d'ordre naturel » (Benazzo, $2004: 45)$ : les événements sont racontés dans l'ordre dans lequel ils ont eu lieu en 
réalité. La structuration du récit est fortement chronologique et linéaire. Pour ce qui est de la jonction propositionnelle, les apprenants se servent pour la plupart de ce que la tradition appelle la parataxe, c'.-àd. des énoncés juxtaposés ou coordonnés, liés dans ce dernier cas par et ou mais. Si le lien sémantique entre plusieurs événements est plus spécifique, souvent causal ou temporel, celui-ci est généralement laissé implicite ; les relations entre ces événements sont marquées notamment par des « moyens indirects, des adverbes, des moyens lexicaux et des relations d'ordre entre séquences » (Véronique, $2005: 14$ ). Ce n'est qu'à un stade plus avancé que les apprenants commencent à produire leurs premières formes de subordination. Il s'agit alors de subordonnées complétives régies par des verbes du type dire (Véronique, 2004) ou de nature aspectuelle comme commencer (Welcomme, 2013), et de certaines adverbiales, notamment les temporelles et les causales (Kirchmeyer, 2002; Benazzo, 2004 ; Welcomme, 2013). Certains apprenants essaient également de produire des relatives introduites par qui ou que.

Lorsque les apprenants développent leur compétence générale en français L2, leurs narrations ne suivent plus nécessairement l'ordre parfaitement chronologique dans lequel ont eu lieu les événements; ils structurent leur discours de plus en plus souvent de manière complexe et non linéaire. Les narrations sont caractérisées à présent par un plus grand nombre d'énoncés bipropositionnels (les énoncés composés de plus de deux propositions demeurent rares). Même si ces énoncés complexes sont souvent construits à partir de jonctions paratactiques, comme aux stades antérieurs, la parataxe perd en importance en faveur de différentes formes de subordination. De ce fait, les relations formelles et sémantiques entre les propositions deviennent plus variées, ce qui est reflété par un ample répertoire de connecteurs différents (Benazzo, 2004; Kerr-Barnes, 1998), dont certains se trouvent même employés au niveau macrosyntaxique, tel parce que comme introducteur de commentaires ou de retours en arrière au niveau discursif (Noyau \& Vasseur, 1986).

Ainsi, au fur et à mesure de la progression en FL2, les subordonnées adverbiales que produisent les apprenants ne sont plus uniquement causales ou temporelles, mais également du type conditionnel ou final (Véronique, 2004). Le répertoire de subordonnées relatives que les apprenants sont capables de produire s'étend aussi : là où ils se limitaient au début aux relatives introduites par qui et, plus rarement, par que, ils emploient maintenant des mots introducteurs comme dont et lequel et associent des rôles syntaxiques différents aux relatives (Welcomme, 2013). Dans le domaine des subordonnées complétives, finalement, les productions des apprenants du français L2 se développent de manière très importante. Aux stades initiaux d'apprentissage, les quelques complétives qu'ils produisent sont introduites par un nombre limité de verbes gouvernants très fréquents (comme vouloir) et comportent presque toujours un verbe fini. Lorsqu'ils deviennent plus compétents en français, les apprenants produisent des complétives à prédicat fini ou non fini, gouvernées par un gamme plus ample de verbes, aspectuels au début (Il commence à chanter) et puis modaux (Il peut chanter, Il doit chanter). Certaines formes de subordination, cependant, demeurent rares même dans les productions des apprenants avancés. Il s'agit des gérondifs et des participiales (Demol \& Hadermann, 2008 ; Degand \& Hadermann, 2009 ; Welcomme, 2013).

Globalement, la compétence croissante des apprenants du français L2 implique une complexification et une hiérarchisation progressives de leur structuration du discours. Ils évoluent d'un emploi très fréquent des modes parataxiques, c'est-à-dire la juxtaposition et la coordination de propositions, à un emploi de plus en plus fréquent d'hypotaxe ou de subordination. Le degré d'intégration caractérisant leur discours augmente donc. Le marquage des liens entre les événements ou les propositions devient plus explicite et plus spécifique. Nous assistons, en outre, à une diversification fonctionnelle : là où les débutants se servent souvent d'un gamme limité de connecteurs, qui remplissent chacun plusieurs fonctions, les apprenants plus avancés élargissent leur répertoire de connecteurs et commencent à associer une fonction spécifique à chacun d'eux (Benazzo, 2004). Le «functional overlap » (Kerr-Barnes, 1998 : 206) tend donc à se réduire de plus en plus.

Sur cette base, plusieurs chercheurs ont proposé ces dernières années des itinéraires acquisitionnels décrivant le développement langagier d'apprenants en français langue seconde (Schlyter, 2003 ; Bartning \& Kirchmeyer, 2003 ; Bartning \& Schlyter, 2004), qui sont basés en partie sur la maîtrise de la jonction propositionnelle. Plus particulièrement, ils distinguent dans le développement des apprenants six stades, identifiés non seulement à partir des mécanismes de jonction, mais également d'autres structures morpho- 
syntaxiques, comme le système temps-mode-aspect, la négation, l'emploi correct ou non des pronoms et des articles, et l'accord en genre entre l'article et son nom tête (Schlyter, $2003: 7$ ).

Le stade initial, comparable selon Bartning \& Schlyter (2004) à la variété prébasique de Klein \& Perdue (1997), est caractérisé par l'emploi de conjonctions coordonnantes (et, mais) ou par la juxtaposition, comprenant souvent le connecteur puis. La production de relations subordonnantes est rare, sauf quelques cas isolés de je voudrais suivi d'un syntagme verbal, que Bartning \& Kirchmeyer (2003) considèrent comme des expressions figées plutôt que comme des réalisations intentionnelles de subordination. Au stade post-initial, correspondant plus ou moins à la variété basique (Klein \& Perdue, 1997), les énoncés ne sont plus toujours monopropositionnels, et les apprenants produisent plusieurs types de subordonnées : des adverbiales circonstancielles introduites par parce que et quand, et des combinaisons d'un verbe modal avec un infinitif, comme il ne veut pas rester (Schlyter, 2003 : 22) ou je peux aller (Bartning \& Kirchmeyer, $2003: 3$ ). Ici apparaissent également les premières tentatives de relativisation avec qui et que. $\mathrm{Au}$ stade intermédiaire, alors, le répertoire propositionnel s'élargit: les adverbiales et les combinaisons modales du stade 2 sont maintenant très fréquentes, tout comme les complétives et les relatives introduites par qui, que, si et où. Au stade suivant, appelé avancé bas (Bartning \& Kirchmeyer, 2003) ou avancé inférieur (Schlyter, 2003), les apprenants introduisent de nouvelles relatives et interrogatives, surtout marquées par ce que et dont. Ils produisent aussi des énoncés comptant plus de deux propositions, et commencent également à employer des subordonnées requérant le subjonctif, notamment il faut que suivi d'une proposition finie. Ce n'est qu'au stade avancé moyen qu'ils ajoutent des «propositions compaquetées » (Schlyter, 2003 : 35) à leur répertoire, c'.-à-d. des gérondifs et des constructions infinitives comme après avoir étudié (Schlyter, 2003 : 37). Ici apparaissent aussi les premiers cas d'ellipse. Les autres différences entre ce stade et le précédent se situent dans d'autres domaines morphosyntaxiques. Le stade avancé supérieur, le dernier avant la maîtrise quasi-native, implique la maîtrise presque parfaite des règles morphosyntaxiques du français, même si l'organisation discursive porte toujours les marques de la langue maternelle des apprenants (Bartning \& Kirchmeyer, 2003). Pour ce qui est de la jonction propositionnelle, cependant, ils atteignent "un haut degré d'empaquetage, d'ellipse et d'intégration des propositions caractérisant une capacité à gérer plusieurs niveaux informationnels au sein du même énoncé » (Bartning \& Schlyter, 2004 : 296).

Le bref tour d'horizon ci-dessus montre que beaucoup d'études sur le sujet sont limitées à l'examen d'une forme ou d'une construction spécifique et, lorsque leur portée est plus globale, elles sont fondées soit sur la bipartition classique coordination/subordination, soit sur la répartition des classes traditionnelles (circonstancielles, complétives, relatives, infinitives...). Or, si les chercheurs ont longtemps été convaincus que l'acquisition de la jonction propositionnelle consistait à passer de la coordination vers la subordination, des recherches récentes remettent ceci en cause (Norris \& Ortega, 2009 ; Welcomme, 2013). En outre, les différentes constructions composant, par exemple, le groupe des circonstancielles ne sont pas maîtrisées au même stade de développement; Jean vient quand Paul part, par exemple, sera acquis bien avant Jean vient bien que Paul parte (Schlyter, 2003). La classe des 'infinitives', elle aussi, contient des structures très diverses, telles Il va parler demain vs. Il espère parler demain, qui ne seront pas nécessairement maîtrisées au même stade en FL2. Finalement, ce qui est traditionnellement appelé coordination peut poser problème tout comme les types de subordination. Ainsi, les cas de la coordination elliptique (Jean a fait la vaisselle et puis nettoyé) s'avèrent beaucoup plus difficile à maîtriser par les apprenants que les cas de coordination sans réduction (Jean a fait la vaisselle et puis il a nettoyé) (Schlyter, 2003 ; Buysse, 2014).

En conclusion, les distinctions traditionnelles des types et modes de jonction ne recouvrent que très partiellement les stades successifs d'acquisition de la liaison de propositions. Une appréhension plus globale du processus de maîtrise de la jonction propositionnelle requiert donc que les paramètres qui fondent la répartition des tours soient affinés sur la base de critères globaux et cohérents. Les propositions de la RRG fournissent un tel cadre. 


\section{La cosubordination comme mode de jonction}

La Grammaire du Rôle et de la Référence (Role and Reference Grammar ou RRG ; Van Valin \& LaPolla, 1997 ; Van Valin, 2005) propose une redistribution des différentes sortes de liaisons propositionnelles à partir d'une tripartition des modes de jonction en coordination, subordination et cosubordination. Ces différents mécanismes de jonction propositionnelle se distinguent essentiellement l'un de l'autre par deux paramètres structurels : la dépendance et l'intégration. La subordination implique l'intégration d'une structure propositionnelle au sein d'une autre en tant qu'argument ou adjoint, comme dans les exemples (1) et (2) ci-dessous :

Il m'a dit qu'il ne travaille jamais le jeudi.

(2) Il ne travaille jamais le jeudi parce qu'il s'occupe de ses petits-enfants.

Dans (1), qu'il ne travaille jamais le jeudi fonctionne comme objet direct contrôlé par Il (m')a dit, tandis que parce qu'il s'occupe de ses petits-enfants dans (2) sert d'adjoint expliquant la cause de la proposition matrice Il ne travaille jamais le jeudi. La subordination peut donc être de deux types; si la partie intégrée du tout fonctionne comme argument, nous parlerons de subordination de type complétif (daughter chez Van Valin \& LaPolla, 1997), tandis que la subordination impliquant un adjoint adverbial sera simplement appelée adverbiale. L'intégration d'une proposition au sein d'une autre est une caractéristique typique de la subordination, et elle implique automatiquement que la structure intégrée est par la même occasion réduite à une position de dépendance vis-à-vis de la structure matrice, sans laquelle elle ne pourrait pas fonctionner. La subordination sera donc définie dans la RRG par les traits [+ intégration] et donc aussi [+ dépendance].

La coordination sera marquée par une valeur négative pour ces deux paramètres. Autrement dit, deux propositions seront liées sans que l'une fonctionne comme argument syntaxique ou comme adjoint adverbial au sein de l'autre et aussi sans qu'elle soit dépendante de l'autre de quelle manière que ce soit. La coordination est donc définie en RRG comme [- intégration], [- dépendance], et pourra y prendre la forme des liaisons illustrées en (3) et en (4) :

(3) Jean est médecin et son épouse a sa propre entreprise de produits biologiques.

(4) Le professeur a dit à Max de se taire.

En outre, pour la RRG, certains types de liaisons n'entrent pas dans le modèle binaire (Van Valin, 2005). Des énoncés comme (5) et (6), notamment, sont généralement considérés comme des cas de subordination, et le second prédicat avec ses arguments est appréhendé comme l'objet direct du verbe principal de la phrase matrice.

(5) Paul veut laver la voiture.

(6) Elle a oublié de signer le document.

Dans les approches traditionnelles, laver la voiture sera analysé comme l'object direct de Paul veut ((5)), à l'instar de ce trophée dans Paul veut ce trophée. Il en est de même pour (de) signer le document, dépendant de Elle a oublié ((6)). Pourtant, ces prétendus objets directs ne fonctionnent pas comme des objets directs nominaux. Pour le démontrer, Van Valin \& LaPolla (1997: 462) utilisent les tests de la passivation et de la transformation en clivée. Ces transformations sont bien permises dans (7)-(8), tandis qu'elles ne sont pas acceptables dans (5)-(6) :

(7) Paul veut ce trophée.

(7)' ? Ce trophée est voulu par Paul.

(7)' C'est ce trophée que Paul veut.

(8) Des spectatrices distraites ont oublié ces manteaux.

(8)' Ces manteaux ont été oubliés par des spectatrices distraites. 
(8)' Ce sont ces manteaux que des spectatrices distraites ont oubliés.

(5) Paul veut laver la voiture.

(5)' * Laver la voiture est voulu par Paul.

(5)", * C'est laver la voiture que Paul veut.

(6) Elle a oublié de signer le document.

(6) * (De) signer le document a été oublié par elle.

(6)" * C'est (de) signer le document qu'elle a oublié.

Selon Van Valin \& LaPolla (1997 : 462), ceci montre que l'on n'a pas affaire dans (5) et (6) à des arguments nucléaires («core arguments ») d'un verbe matrice, et que ces structures ne se trouvent donc pas dans une relation subordonnée vis-à-vis du prédicat central. Cependant, il ne s'agit pas non plus de cas de coordination : laver la voiture dans (5) dépend effectivement de $I l$ veut d'une certaine manière, et ceci vaut également pour l'exemple (7). Il y a donc bien une certaine dépendance, qui sera analysée dans ce qui suit comme une forme de dépendance d'opérateur(s) ou d'argument(s). Van Valin \& LaPolla (1997) et Van Valin (2005) introduisent dès lors un troisième mode de liaison, qu'ils appellent la cosubordination. Elle sera définie comme [- intégration], [+ dépendance].

La dépendance qui caractérise la cosubordination n'est cependant pas du même genre que celui résultant de l'intégration qui caractérise la subordination. La structure dépendante est soumise dans ce cas-ci à une dépendance d'opérateurs et/ou à une dépendance d'arguments. La dépendance d'opérateurs signifie que la structure dépendante manque au moins un opérateur grammatical. Cet opérateur n'est explicité qu'au sein de la matrice, et il faut donc avoir recours à ce dernier pour saisir la structure dépendante. Dans (6), Paul veut laver la voiture, par exemple, laver la voiture est de forme non finie, c'est-à-dire que le nombre et la personne du prédicat verbal ne sont pas explicitement indiqués. Ceci vaut également pour son marquage de temps. Afin de remplir ces vides dans la compréhension de l'énoncé, le lecteur ou l'interlocuteur doit avoir recours à la structure matrice, Paul veut, qui transmet son propre marquage de temps, de nombre et de personne au prédicat dépendant. Cet énoncé est également caractérisé par ce que nous appellerons la dépendance d'arguments : le sujet sous-entendu du verbe dépendant est Paul, mais cet argument dépendant n'est pas explicité au sein de la structure dépendante. Il doit, lui aussi, être emprunté à la structure matrice. Il en est de même pour (7), Elle a oublié de signer le document. Le verbe dépendant, signer, ne porte pas de marquage de nombre, de personne ou de temps, mais dépend du verbe de la proposition matrice, a oublié, pour toutes ces spécifications. La dépendance d'argument(s) se manifeste par l'absence du sujet dans la structure signer le document, qu'elle a en commun avec la structure matrice et qu'elle doit donc lui emprunter.

Les trois modes de jonction identifiés par la RRG peuvent être implémentées à trois niveaux structurels différents au sein de la proposition. C'est en particulier le cas de la cosubordination, qui constitue l'axe central de cette contribution. Nous présentons ci-dessous des occurrences de ce mode de jonction aux trois niveaux structurels distingués.

Le niveau de base ou nucléaire est celui du noyau prédicatif. L'établissement d'une jonction cosubordonnante au niveau nucléaire implique que deux prédicats se trouvent fusionnés en un seul prédicat complexe. Les prédicats concernés partagent leurs marqueurs de temps, de nombre et de personne (dépendance d'opérateurs) et prennent un seul ensemble d'arguments primaires (dépendance d'arguments). La cosubordination nucléaire est illustrée par (9) et (10).

(9) Je ferai manger les spaghettis à mon frère.

(10) Il a mis les couverts sur la table.

Dans (9), le prédicat complexe ferai manger est accompagné par ses arguments primaires je (sujet) et les spaghettis (objet direct). L'exemple (10), à son tour, montre que les composantes du prédicat complexe ne doivent pas nécessairement être juxtaposées et qu'un prédicat n'est pas forcément de nature verbale. 
Dans ce cas-ci, a mis sur la table est le nouveau prédicat complexe prenant $i l$ comme sujet et les couverts comme objet direct.

Le mode de liaison peut se situer au niveau de la cellule, composée du prédicat et de ses arguments primaires. Il s'agit alors du niveau cellulaire. Les structures combinées dans ce cas-ci sont des prédicats ayant chacun leur propre ensemble d'arguments, même si un d'eux est généralement partagé par tous les prédicats (dépendance d'arguments). Tout comme au niveau nucléaire, la structure dépendante au niveau cellulaire doit relier sur la structure matrice pour le marquage explicite du nombre, de la personne et du statut temporel (dépendance d'opérateurs).

(5) Paul veut laver la voiture.

(6) Elle a oublié de signer le document.

Dans (5), laver la voiture a besoin de la matrice Paul veut pour l'expression de son sujet sous-entendu et des informations grammaticales associées aux prédicats, c'est-à-dire le temps, le nombre et la personne. Il en est de même pour (de) signer le document dans (6), qui a besoin de la présence de Elle a oublié pour l'expression de son sujet et de toutes ses associations verbales.

Finalement, la liaison peut se situer au niveau clausal. Les prédicats liés y gardent non seulement leurs arguments primaires respectifs, mais également des arguments secondaires et d'éventuels ajouts périphériques. La cosubordination implique alors que l'une des propositions est dépendante de l'autre pour l'expression de ses spécifications verbales (ou du verbe entier, comme dans (11)) ou d'au moins un de ses arguments (le sujet dans (12), par exemple).

(11) Elle dort, et sa fille aussi.

(12) Il a éteint la lumière et fermé la porte.

Au niveau clausal, la proposition dépendante en cosubordination adopte également la force illocutoire de la matrice, comme montré par (13)-(13')'. L'on ne peut pas questionner la clause dépendante sans questionner l'énoncé complet. Dans (13)', par contre, la relation syntaxique est coordonnante au lieu de cosubordonnante, et permet bien aux clauses combinées d'avoir chacune sa propre force illocutoire.

(13) Nathalie a salué Georges mais ignoré Diane. (affirmatif)

(13)’ *Nathalie a salué Georges (affirmatif), mais ignoré Diane ? (interrogatif)

(13)' Nathalie a salué Georges (affirmatif), mais elle a ignoré Diane ? (interrogatif)

La tripartition proposée introduit donc une redistribution importante des structures liées à partir d'une série de paramètres cohérents et généraux. Notre recherche vise à évaluer si cette nouvelle répartition proposée par la RRG, basée sur les modes de jonction et le niveau d'implémentation, permet de mieux saisir le processus de développement de la jonction propositionnelle d'apprenants de français L2. Nous nous centrerons dans cette contribution plus spécifiquement sur l'apport du mode de jonction cosubordonnant.

\section{L'apport de la cosubordination à l'appréhension du développement de la jonction en FL2}

La pertinence de cette tripartition sera évaluée en analysant son apport pour l'analyse de la maîtrise progressive de la liaison propositionnelle dans la production en FL2 d'apprenants néerlandophones. Nous nous appuierons sur les hypothèses formulées par la RRG concernant l'acquisition de L1, que nous détaillerons ci-dessous, pour vérifier leur pertinence dans le domaine de l'appropriation d'une L2.

3.1. Les données proviennent d'un projet plus vaste sur la jonction propositionnelle en français et en anglais L2 (Buysse \& De Clercq, 2014 ; Buysse, 2014 ; Buysse, 2015). Il s'agit d'adolescents, âgés de 12 à 18 ans, vivant en Flandre (la partie néerlandophone de la Belgique) et parlant tous le néerlandais comme langue maternelle. Ces adolescents apprennent le français ainsi que l'anglais à l'école secondaire en tant 
que langues secondes ou étrangères, bien que la présente contribution se limite à la partie française du corpus. Les apprenants ont été subdivisés en quatre groupes de 25 personnes selon leur niveau global de compétence en langue seconde $(\mathrm{n}=100$; Buysse \& De Clercq, 2014). Nous avons ajouté à ce corpus d'apprenants un groupe de la même taille $(n=25)$ de locuteurs natifs du français, eux aussi des adolescents, fonctionnant comme groupe de contrôle. Les données linguistiques produites par les participants sont des narrations orales sur base de dessins sans texte (Frog, where are you? ; Mayer 1969), permettant une production relativement libre, mais en même temps contrainte par la trame de l'histoire de base et par les mêmes indices visuels proposés à chaque participant. Ces narrations ont été transcrites et analysées manuellement, et la nature sémantique et syntaxique de chaque cas de jonction propositionnelle a été indiquée. La validité statistique des tendances dégagées des données a été contrôlée par le biais du programme SPSS 22 (IBM, 2013).

3.2. Van Valin (2001) décrit le développement de la jonction propositionnelle chez de jeunes enfants acquérant leur langue maternelle, basée sur des observations d'études en acquisition de diverses L1. Il déduit de ces observations un nombre de principes généraux au sujet de l'acquisition progressive des mécanismes de jonction, dont certains impliquent directement le phénomène de la cosubordination.

Il soutient en particulier que les premières formes de jonction à apparaître dans le discours des enfants acquérant leur L1 sont de nature non subordonnante. Autrement dit, la coordination et la cosubordination, telles qu'elles sont définies en RRG, apparaissent à un stade plus précoce dans les narrations de ces enfants, avant toute forme de subordination. Ceci serait dû à l'intégration syntaxique, qui caractérise la subordination mais est absente des deux autres formes de jonction, et qui serait relativement difficile à maîtriser et à (re)produire pour les jeunes enfants en début d'acquisition.

A l'intérieur de l'ensemble des jonctions non subordonnantes, celles qui n'impliquent donc pas d'intégration d'une structure au sein d'une autre, les liens cosubordonnants devraient être plus faciles à maîtriser et à produire que la coordination (Van Valin, 2001). Ceci est reflété par l'apparition précoce de la cosubordination dans les narrations en L1 des jeunes enfants. Selon Van Valin (2001), cet ordre d'apparition est lié à la dépendance d'opérateurs et d'arguments qui distingue la cosubordination des autres formes de jonction. La combinaison de structures partageant certains éléments, dont des arguments du prédicat ou des opérateurs grammaticaux comme le temps, le nombre et la force illocutoire, serait relativement facile à conceptualiser et à reproduire ensemble dans une construction complexe. Ceci faciliterait donc la production des jonctions cosubordonnantes. Dans les cas de coordination, par contre, les structures combinées pourraient en principe fonctionner indépendamment au niveau structurel pertinent, et aucun argument ou opérateur typique de ce niveau n'est obligatoirement partagé. Le locuteur doit donc prendre en considération un nombre plus élevé de composantes et d'opérateurs en construisant une jonction complexe selon les normes de sa langue cible.

3.3. La présente contribution veut vérifier si ces constats rapportés dans Van Valin (2001) s'appliquent non seulement à l'acquisition d'une langue maternelle, mais également au processus d'apprentissage d'une L2, en l'occurrence ici du français en tant que langue seconde. L'examen sera basé sur les deux questions de recherche suivantes :

(1) Les jonctions non subordonnantes (c'est-à-dire [- intégration]) sont-elles proportionnellement plus nombreuses au début du processus d'acquisition qu'à la fin du processus observé, au contraire des jonctions comportant une subordination [+ intégration], qui seraient proportionnellement moins nombreuses au début du processus d'acquisition qu'à sa fin ?

Une réponse positive à cette question de recherche fournirait des indices pertinents tendant à confirmer qu'en acquisition d'une L2 également, comme dans le cas des jeunes enfants acquérant leur L1, les jonctions non subordonnantes ([- intégration]) sont acquises avant les jonctions comportant une subordination ([+ intégration]).

Nous décrirons l'ordre d'acquisition relative des jonctions subordonnantes par rapport aux coordonnantes et aux cosubordonnantes, et nous comparerons également les productions des apprenants du français L2 à celles d'un groupe d'adolescents locuteurs natifs du français. 
(2) Au sein du groupe des jonctions sans intégration, les jonctions cosubordonnantes ([+ dépendance]) sont-elles proportionnellement plus nombreuses au début du processus d'acquisition observé ici qu'à la fin, et les jonctions coordonnantes ([- dépendance]) sont-elles proportionnellement moins nombreuses au début de ce processus qu'à la fin de celui-ci ?

Si nos données suggèrent une réponse positive à cette question de recherche, ceci pourrait confirmer qu'en acquisition d'une langue seconde, tout comme dans le cas des enfants en acquisition de leur langue maternelle, la cosubordination ([- intégration], [+ dépendance $]$ ) est acquise avant la coordination ([intégration], [- dépendance]).

La section suivante décrit le développement de la jonction propositionnelle dans la production des 4 groupes d'apprenants néerlandophones de FL2 et compare celle-ci à celle du groupe de contrôle.

\section{Description des données et commentaires}

A partir de la description du développement des différents mécanismes de jonction propositionnelle dans notre corpus, nous nous concentrerons plus spécifiquement sur la cosubordination, et nous comparerons cet emploi avec celui des locuteurs natifs. Sur base de ces informations, nous essayerons de répondre aux deux questions de recherche exposées dans la section 3.

Le Tableau 1 fournit le taux d'occurrence moyen de chaque mécanisme de jonction au sein de notre corpus, pour les quatre niveaux d'apprenants (1-4) et pour le groupe de contrôle composé de locuteurs natifs du français $(\mathrm{N})$.

Tableau 1 : Pourcentage moyen des formes de jonction dans le corpus

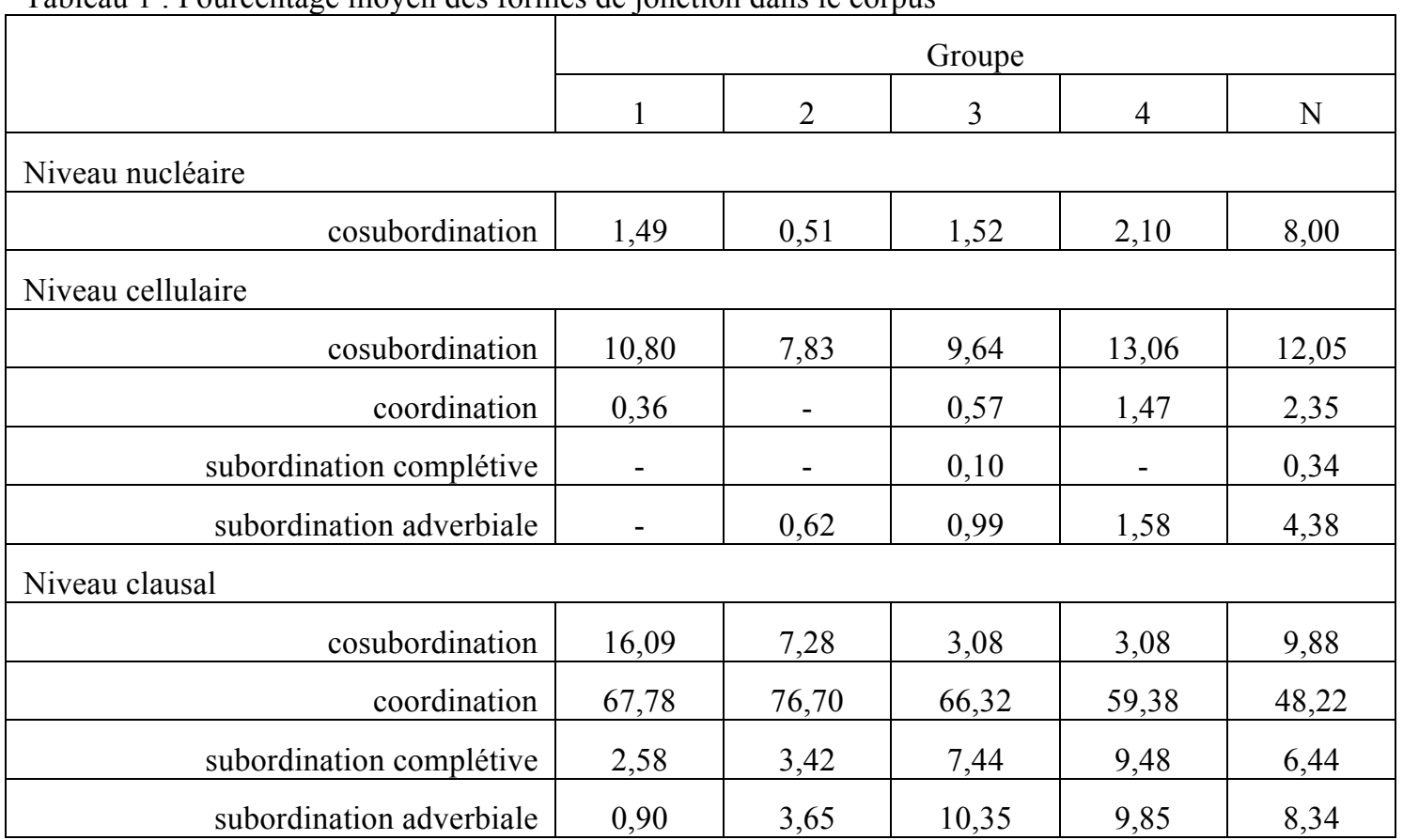

Ce tableau montre l'évolution de l'emploi des différents modes de jonction propositionnelle dans les quatre groupes d'apprenants de notre corpus.

Certains mécanismes de jonction sont produits le plus fréquemment par les apprenants moins avancés des groupes 1 et 2 et reculent ensuite en fréquence lorsque les apprenants deviennent plus compétents en FL2. Ceci vaut, en particulier, pour la cosubordination et la coordination au niveau clausal. Les apprenants du 
groupe 1 , le moins avancé, se distinguent de manière statistiquement significative $(p=0,003)$ de ceux plus avancés des groupes 3 et 4 dans leur emploi de la cosubordination clausale. Dans le cas de la coordination clausale, aucune différence significative ne peut être observée entre les quatre groupes d'apprenants, mais les taux de production moyen montrent que son emploi recule surtout après le groupe 2 .

D'autres formes de jonction, par contre, figurent rarement dans les productions des apprenants débutants, mais gagnent en fréquence lorsque la compétence générale des apprenants s'accroît. La coordination au niveau cellulaire, par exemple, est nettement plus importante dans les productions des groupes 3 et 4 que dans celles des groupes 1 et 2 , ce qui est confirmé par les analyses statistiques ( $p=0,000)$; elle est même absente de ce dernier groupe. La subordination adverbiale cellulaire, à son tour, est complètement absente des narrations du groupe 1 et devient de plus en plus productive aux niveaux plus avancés, même si l'évolution n'est pas d'une telle importance qu'elle occasionne une différence statistiquement significative. Il en est de même pour les deux formes de subordination au niveau clausal. La subordination complétive gagne en fréquence, de manière linéaire, lorsque les apprenants deviennent plus compétents, et la même tendance vaut pour les trois premiers groupes d'apprenants dans le cas de son pendant adverbial. Le taux d'usage de ce mode de jonction diminue à nouveau légèrement dans le groupe 4, mais demeure clairement en dessous de celui des apprenants moins avancés. Dans ces deux catégories, les apprenants des groupes 1 et 2 se distinguent de manière statistiquement significative de ceux des groupes 3 et $4(\mathrm{p}=0,000)$.

La fréquence d'emploi de la cosubordination, au niveau nucléaire ainsi que cellulaire, finalement, fluctue entre les groupes d'apprenants. Dans les deux cas, le mode de jonction atteint son taux d'usage le plus bas dans le groupe 2 et le plus élevé dans le groupe 4, mais aucune différence statistiquement significative ne peut être observée entre les groupes d'apprenants. La subordination complétive au niveau cellulaire est tellement rare dans notre corpus - un cas produit par un apprenant du groupe 3 et une poignée de cas chez les locuteurs natifs - qu'elle n'induit aucun effet statistique.

Les taux d'utilisation des différents modes de jonction propositionnelle auprès des apprenants diffèrent régulièrement de celui des locuteurs natifs. Dans les cas de la cosubordination nucléaire $(p=0,000)$, de la coordination cellulaire $(p=0,016)$ et de la subordination adverbiale cellulaire $(p=0,000)$, même les apprenants les plus avancés se servent moins fréquemment de ces modes de jonction que les locuteurs natifs ; dans le cas de la cosubordination clausale $(\mathrm{p}=0,003)$, la même tendance vaut pour les apprenants avancés, mais les moins avancés produisent plus de cas de cette jonction que le groupe de contrôle. Les occurrences de coordination clausale, à leur tour, révèlent un suremploi de ces mécanismes chez les apprenants par rapport aux locuteurs natifs du groupe de contrôle $(p=0,000)$. Dans leur emploi de la cosubordination cellulaire et les deux formes de subordination clausale, finalement, les apprenants plus avancés des groupes 3 et 4 se comportent de manière semblable aux locuteurs natifs.

Quelles réponses ces données offrent-elles aux questions de recherche?

(1) Les jonctions non subordonnantes (c'est-à-dire [- intégration]) sont-elles proportionnellement plus nombreuses au début du processus d'acquisition qu'à la fin du processus observé, au contraire des jonctions comportant une subordination [+ intégration], qui seraient proportionnellement moins nombreuses au début du processus d'acquisition qu'à sa fin ?

Dans nos observations, nous distinguerons trois formes de comparaison différentes. Nous regrouperons d'abord toutes les jonctions non subordonnantes, à tous les niveaux structurels, d'une part et toutes les jonctions subordonnantes d'autre part. Ensuite, nous comparerons les modes de jonction non subordonnantes et subordonnantes au niveau structurel cellulaire, et finalement nous procéderons de la même manière au niveau clausal. 
Tableau 2 : Jonctions non subordonnantes vs. jonctions subordonnantes

\begin{tabular}{|r|c|c|c|c|}
\hline \multirow{2}{*}{} & \multicolumn{5}{|c|}{ Groupe } \\
\cline { 2 - 5 } & 1 & 2 & 3 & 4 \\
\hline non subordonnantes & 96,52 & 92,32 & 81,13 & 79,09 \\
\hline subordonnantes & 3,48 & 7,69 & 18,88 & 20,91 \\
\hline
\end{tabular}

Ce tableau oppose toutes les jonctions non subordonnantes (cosubordination nucléaire, cosubordination cellulaire, coordination cellulaire, cosubordination clausale et coordination clausale) à l'ensemble des jonctions subordonnantes (subordination cellulaire complétive et adverbiale et subordination clausale complétive et adverbiale).

Il montre que, dans les narrations de tous les groupes d'apprenants, les jonctions non subordonnantes sont nettement plus fréquentes que celles comportant une subordination. Au fur et à mesure que les apprenants deviennent plus compétents en FL2, cependant, la proportion de non subordonnantes se réduit et celle de la subordination s'accroît. La différence, confirmée par les analyses statistiques, se situe notamment entre les groupes 1 et 2 d'une part et les groupes 3 et 4 d'autre part $(p=0,000)$. Globalement, les jonctions non subordonnantes semblent donc effectivement être proportionnellement plus nombreuses au début et moins nombreuses à la fin du développement tel que le reflètent nos données, tandis que le mouvement inverse s'applique aux deux formes de subordination (complétive et adverbiale).

Tableau 3: Jonctions cellulaires non subordonnantes vs. jonctions cellulaires subordonnantes (proportions par rapport au nombre total de jonctions)

\begin{tabular}{|r|c|c|c|c|}
\hline & \multicolumn{5}{|c|}{ Groupe } \\
\cline { 2 - 5 } & 1 & 2 & 3 & 4 \\
\hline non subordonnantes & 11,16 & 7,83 & 10,21 & 14,53 \\
\hline subordonnantes & - & 0,62 & 1,09 & 1,58 \\
\hline
\end{tabular}

Le Tableau 3 oppose les jonctions cellulaires non subordonnantes ([- intégration], c'est-à-dire la cosubordination et la coordination cellulaires, à la subordination complétive et adverbiale au même niveau structurel. Les chiffres représentent le pourcentage d'emploi de la jonction donnée au sein de l'ensemble de jonctions complexes.

A nouveau, la dominance des modes de connexion non subordonnants est claire : les liens non intégrants sont plus fréquents dans les narrations de chaque groupe d'apprenants que ceux comportant une intégration En accord avec notre hypothèse, la proportion de subordonnantes cellulaires s'accroît lentement mais sûrement lorsque les apprenants deviennent plus compétents en FL2. Dans la catégorie non subordonnante, par contre, l'hypothèse n'est pas confirmée : au lieu de perdre en importance dans les groupes d'apprenants plus avancés, le taux d'emploi des liens non subordonnants fluctue fortement à travers le corpus L2. Il atteint son niveau le plus bas dans le group 2 pour remonter ensuite et atteindre le taux le plus élevé dans le groupe 4.

Tableau 4 : Jonctions clausales non subordonnantes vs. jonctions clausales subordonnantes (proportions par rapport au nombre total de jonctions)

\begin{tabular}{|r|c|c|c|c|}
\hline \multirow{2}{*}{} & \multicolumn{4}{|c|}{ Groupe } \\
\cline { 2 - 5 } & 1 & 2 & 3 & 4 \\
\hline non subordonnantes & 83,87 & 83,98 & 69,40 & 62,46 \\
\hline subordonnantes & 3,48 & 7,07 & 17,79 & 19,33 \\
\hline
\end{tabular}


Le Tableau 4 oppose les jonctions clausales non subordonnantes (cosubordination et coordination) d'une part et les deux formes de subordination clausale d'autre part. Comme dans les autres tableaux, les chiffres représentent la proportion d'usage du mode de jonction donné par rapport au total de jonctions employées dans le corpus.

En accord avec l'hypothèse basée sur Van Valin (2001), les jonctions subordonnantes sont peu fréquentes dans les narrations des groupes les moins compétents et deviennent de plus en plus productives lorsque leur compétence en FL2 s'accroît. Les liens non subordonnants subissent l'évolution inverse : ils perdent graduellement en importance en faveur des deux types de subordination clausale, même s'ils demeurent globalement beaucoup plus fréquents que la subordination.

Au niveau cellulaire tout comme au niveau clausal, les jonctions subordonnantes ([+ intégration]) sont donc effectivement proportionnellement moins nombreuses au début du processus d'acquisition FL2 selon nos données qu'à la fin, en accord avec la première hypothèse de la RRG (Van Valin, 2001). Les jonctions non subordonnantes ([- intégration]), à leur tour, présentent la tendance inverse au niveau global : elles sont plus fréquentes dans les narrations des apprenants moins avancés que dans celles des plus compétents. Si l'on regarde les différents niveaux structurels qui permettent la comparaison entre les jonctions subordonnantes et non subordonnantes, par contre, ce ne sont que les jonctions clausales qui confirment cette tendance de décroissance graduelle. Au niveau cellulaire, plutôt que de perdre en importance lorsque la compétence des apprenants s'accroît, le taux d'usage des non subordonnantes fluctue de manière non systématique. En général, cependant, nos données semblent avancer de forts indices pour confirmer la première hypothèse de Van Valin (2001). Bref, les mêmes principes de répartition entre non-subordination et subordination semblent être à l'œuvre dans l'acquisition du français L2 que dans l'acquisition en L1.

(2) Au sein du groupe des jonctions sans intégration, les jonctions cosubordonnantes ([+ dépendance]) sont-elles proportionnellement plus nombreuses au début du processus d'acquisition observé ici qu'à la fin, et les jonctions coordonnantes ([- dépendance]) sont-elles proportionnellement moins nombreuses au début de ce processus qu'à la fin de celui-ci ?

Tableau 5 : Cosubordination vs. coordination

\begin{tabular}{|r|c|c|c|c|}
\hline \multirow{2}{*}{} & \multicolumn{5}{|c|}{ Groupe } \\
\cline { 2 - 5 } & 1 & 2 & 3 & 4 \\
\hline cosubordination & 28,38 & 15,62 & 14,24 & 18,24 \\
\hline coordination & 68,14 & 76,70 & 66,89 & 60,85 \\
\hline
\end{tabular}

Le Tableau 5 ci-dessus oppose l'ensemble des jonctions cosubordonnantes (aux niveaux nucléaire, cellulaire et clausal) à l'ensemble des jonctions coordonnantes (aux niveaux cellulaire et clausal). Selon les données du corpus, les jonctions cosubordonnantes, perdent graduellement en fréquence du groupe 1 au groupe 3. Même si les apprenants les plus avancés produisent à nouveau un peu plus de jonctions de ce type, la tendance correspond globalement aux prédictions de la RRG. L'usage global de la coordination par les apprenants de notre corpus est nettement plus important que l'emploi de la cosubordination. La fréquence de ses occurrences tend globalement à diminuer du groupe 1 au groupe 4, bien que ce mouvement ne soit pas linéaire : le taux d'emploi de la coordination augmente initialement entre les groupes 1 et 2, pour diminuer ensuite. Cette évolution globale ne correspond pas à la prédiction de la RRG. Dans ce qui suit, nous regarderons de plus près les évolutions dans l'emploi de la cosubordination et de la coordination aux niveaux structurels où elles s'opposent. 
Tableau 6: Cosubordination vs. coordination cellulaires (proportions par rapport au nombre total de jonctions)

\begin{tabular}{|r|c|c|c|c|}
\hline \multirow{2}{*}{} & \multicolumn{4}{|c|}{ Groupe } \\
\cline { 2 - 5 } & 1 & 2 & 3 & 4 \\
\hline cosubordination & 10,80 & 7,83 & 9,64 & 13,06 \\
\hline coordination & 0,36 & - & 0,57 & 1,47 \\
\hline
\end{tabular}

Au niveau cellulaire, le taux d'emploi des jonctions cosubordonnantes fluctue à travers l'ensemble du corpus FL2. Ce sont les apprenants les plus avancés, du groupe 4, qui produisent le taux le plus élevé de cosubordinations cellulaires, suivis dans l'ordre par les groupes 1, 3 et 2. Aucune évolution linéaire ne peut donc être décelée à partir de ces observations. La coordination au même niveau structurel, par contre, tend à devenir de plus en plus importante dans les productions de nos apprenants, ce qui correspond aux prédictions, même si sa proportion demeure à tout temps limitée et si elle est temporairement absente des productions du groupe 2 .

Tableau 7: Cosubordination vs. coordination clausales (proportions par rapport au nombre total de jonctions)

\begin{tabular}{|r|c|c|c|c|}
\hline \multirow{2}{*}{} & \multicolumn{4}{|c|}{ Groupe } \\
\cline { 2 - 5 } & 1 & 2 & 3 & 4 \\
\hline cosubordination & 16,09 & 7,28 & 3,08 & 3,08 \\
\hline coordination & 67,78 & 76,70 & 66,32 & 59,38 \\
\hline
\end{tabular}

Au niveau clausal, les jonctions cosubordonnantes sont nettement moins fréquentes que celles comportant de la coordination, à n'importe quel niveau de compétence en FL2. Cependant, le taux d'utilisation de la cosubordination clausale perd en fréquence, de manière statistiquement significative $(p=0,003)$, lorsque les apprenants deviennent plus compétents en FL2. La différence se situe entre les groupes 1 et 2 d'une part et 3 et 4 d'autre part. La coordination clausale, elle aussi, perd en importance du groupe 1 au groupe 4, mais contrairement à la cosubordination au même niveau, cette évolution n'est pas linéaire : le taux d'utilisation de cette forme de jonction est le plus élevé dans la production des apprenants du groupe 2. Si l'évolution de la cosubordination correspond aux hypothèses, ce n'est pas le cas de celle de la coordination.

La deuxième hypothèse de Van Valin (2001), qui postule que la cosubordination ([+ dépendance]) est plus productive aux stades précoces d'acquisition tandis que la coordination ([- dépendance]) devient plus importante aux stades plus avancés, ne semble donc que partiellement confirmée par nos données. Au niveau cellulaire, la coordination devient effectivement plus productive lorsque les apprenants de FL2 deviennent plus compétents, et la cosubordination est très fréquente à partir du premier niveau de compétence; pourtant, cette cosubordination cellulaire ne montre pas l'évolution décroissante attendue sur base de Van Valin (2001). Au niveau clausal, par contre, la cosubordination - fréquente dans les groupes de compétence limitée - perd effectivement en importance de manière significative, mais cette fois-ci, c'est la coordination qui ne répond pas à l'hypothèse : à tous les stades, elle est beaucoup plus fréquente que la cosubordination, et au lieu de devenir globalement plus fréquente lorsque les apprenants deviennent plus compétents, elle perd en importance.

\section{Conclusions}

Dans ce qui précède, nous avons proposé le concept de la cosubordination comme il est défini par Van Valin (2005) en tant qu'alternative à la dichotomie traditionnelle entre la coordination et la subordination. 
La présente étude s'était posé comme objectif d'évaluer si l'identification d'un mode de jonction particulier, la cosubordination, et la redistribution des constructions sur la base d'une tripartition cosubordination/subordination/coordination permet de mieux saisir le processus d'appropriation de la jonction propositionnelle en FL2. Pour ce faire, nous avons formulé deux questions de recherche centrées sur le rôle de la cosubordination, basées sur des hypothèses posées par Van Valin (2001) dans un contexte d'acquisition de L1.

En premier lieu, les tendances prédites par Van Valin (2001) sont plutôt confirmées par nos données en FL2. Les apprenants du français L2 semblent effectivement maîtriser plus facilement les modes de jonction non subordonnants que ceux impliquant une forme de subordination (et donc d'intégration) De même, le taux d'emploi des mécanismes de subordination tend à augmenter avec le niveau de maîtrise. A l'intérieur du groupe non subordonnant, la cosubordination semble globalement plus facile à maîtriser par les apprenants les moins avancés que la coordination, ce qui est en particulier vrai au niveau structurel de la clause; au niveau cellulaire, c'est la coordination qui progresse à travers les groupes, même si le taux d'emploi reste très limité par rapport aux autres catégories. La dominance quantitative de la coordination dans l'ensemble du corpus s'explique sans doute par la nature orale et narrative de la tâche à la base de la production des apprenants. Globalement donc, les apprenants du FL2 se comportent de manière assez semblable aux jeunes enfants d'origines diverses dont Van Valin (2001) décrit le processus d'acquisition en L1, et les principes généraux de jonction semblent s'appliquer aussi bien à celui-ci qu'à l'apprentissage plus tardif d'une langue seconde ou étrangère. La question de savoir si la conclusion de la présente étude vaut pour n'importe quelle L2 mérite évidemment de plus amples recherches.

En outre, la tripartition que fait la RRG entre la coordination, la cosubordination et la subordination permet d'isoler et d'identifier des zones problématiques pour l'acquisition d'une langue seconde, qui ne sont pas toujours saisies par les études traditionnelles. Celles-ci classent, par exemple, des énoncés comme Paul veut laver la voiture et Elle a oublié de signer le document comme des cas de subordination, ce qui ne permet pas de faire la distinction entre ces exemples et des énoncés comme Paul veut que Jean lave la voiture ou Elle pense que sa fille est malade, qui apparaissent bien plus tardivement dans les narrations d'apprenants du français L2. La distinction entre la subordination et la cosubordination, par contre, permet de les séparer et de classer les premiers dans cette dernière catégorie, qui est définie comme [- intégration] mais [+ dépendance], ce qui fournit par la même occasion une explication pour le fait qu'ils sont effectivement acquis - selon Van Valin (2001) et selon la présente contribution - avant les constructions subordonnées classiques. La RRG contribue à l'établissement d'un nouveau modèle de jonction propositionnelle en ne se limitant pas au niveau de la proposition ou de la clause entière, mais en reconnaissant également des mécanismes de complexification propositionnelle aux niveaux plus restreints du noyau et de la cellule. Ainsi, la coordination au niveau cellulaire - difficile à maîtriser pour les apprenants moins avancés dans notre corpus, et devenant plus importante dans leurs narrations lorsqu'ils deviennent plus compétents en FL2 - sera distinguée par la RRG du même mode de jonction au niveau clausal, que les apprenants les moins compétents produisent très fréquemment. De même, la cosubordination clausale est traitée différemment par les apprenants que celle aux niveaux nucléaire et cellulaire. Tandis que la cosubordination nucléaire devient globalement plus importante au fur et à mesure que les apprenants gagnent en compétence en français, la cosubordination au niveau clausal est la plus fréquente parmi les apprenants moins avancés et perd clairement en importance dans les groupes plus compétents.

La tripartition suggérée par la RRG sur la base des critères de dépendance et d'intégration, appliqués à trois niveaux de structuration de la proposition, paraît donc fructueuse pour mieux discerner certaines tendances générales sur le plan de l'acquisition des modes de jonction propositionnelle et pour affiner l'appréhension du développement de la maîtrise de constructions spécifiques. Il nous semble donc que le modèle de jonction propositionnelle avancé par la RRG offre un cadre d'analyse valable pour les études en acquisition de langues secondes, ce que nous venons d'illustrer pour le français L2. L'application de ce nouveau modèle de jonction à des productions de nature diverse, produite en langue seconde par des apprenants de L1 différentes, permettra de vérifier la pertinence plus générale du paradigme proposé en RRG. 


\section{Références bibliographiques}

Bartning, I. \& Kirchmeyer, N. (2003). Le développement de la compétence textuelle à travers les stades acquisitionnels en français L2. Acquisition et interaction en langue étrangère, 19: Les énoncés complexes et leur développement dans l'acquisition des langues, 9-39.

Bartning, I. \& Schlyter, S. (2004). Itinéraires acquisitionnels et stades de développement en français L2. French Language Studies, 14, 281-299.

Benazzo, S. (2004). L'expression de la causalité dans le discours narratif en français L1 et L2. Langages, 38/155, 3351.

Bronckart, J.-P. \& Schneuwly, B. (1984). La production des organisateurs textuels chez l'enfant. Dans Moscato, M. \& Piéraut-Le Bonnec, G. (éds), Le langage. Construction et actualisation. Rouen : Presses Universitaires de Rouen, 165-178.

Buysse, M. (2014). Hiérarchie des relations syntaxiques interprédicationnelles et appropriation en français L2. Dans Neveu, F., Blumenthal, P., Hriba, L., Gerstenberg, A., Meinschaefer, J. \& Prévost, S. (éds), Actes du 4e Congrès Mondial de Linguistique Française, 1419-1435.

Buysse, M. (2015). Hiérarchie des relations sémantiques interprédicationnelles et appropriation en français L2. Círculo de Lingüistica Aplicada a la Comunicación, 63, 97-121.

Buysse, M. \& De Clercq, B. (2014). Matching the data: developing a multilingual corpus of language development. Dans Née, E., Daube, J.-M., Valette, M. \& Fleury, S. (éds), Actes des 12es Journées Internationales d'Analyse Statistique des Données Textuelles, 529-538.

Clark, E.V. (1998). Lexique et syntaxe dans l'acquisition du français. Langue française, 118, 49-60.

Degand, L. \& Hadermann, P. (2009). Structure narrative et connecteurs temporels en français langue seconde. Dans Havu, E., Härmä, J., Helkkula, M., Larjavaara, M. \& Tuomarla, U. (éds), La langue en contexte. Actes du colloque "Représentations du sens linguistique IV», Helsinki 28-30 mai 2008. Helsinki: Société Néophilologique, 19-34.

Demol, A. \& Hadermann, P. (2008). An exploratory study of discourse organisation in French L1, Dutch L1, French L2 and Dutch L2 written narratives. Dans Gilquin, G., Papp, S. \& Diéz-Bedmaer, M.B. (éds), Linking up contrastive and learner corpus research. Amsterdam/Atlanta : Rodopi, 255-282.

Granfeldt, J. \& Nugues, P. (2007). Evaluating stages of development in second language French: A machine-learning approach. Dans Nivre, J., Kaalep, H.-J., Muischnek, K. \& Koit, M. (éds), Proceedings of the 16th Nordic Conference of Computational Linguistics NODALIDA-2007. Publisher ?, 73-80.

IBM Corp. (2013). IBM SPSS Statistics for Mac, Version 22.0. Armonk (NY) : IBM Corp.

Kern, S. (2000). Junction and segmentation in French children's narratives. Psychology of Language and Communication, 4 (1), 47-63.

Kerr-Barnes, B. (1998). The acquisition of connectors in French L2 narrative discourse. Journal of French Language Studies, 8 (2), 189-208.

Norris, J. \& Ortega, L. (2009). Towards an organic approach to investigating CAF in instructed SLA: The case of complexity. Applied Linguistics, 30, 555-578.

Van Valin, R. D. Jr. (2001). The acquisition of complex sentences: A case study in the role of theory in the study of language development. Chicago Linguistic Society Parasession, 36, 511-531.

Van Valin, R. D. Jr. (2005). Exploring the syntax-semantics interface. Cambridge (MA) : Cambridge University Press.

Van Valin, R. D. Jr. \& LaPolla, R. J. (1997). Syntax: Structure, meaning and function. Cambridge (MA) : Cambridge University Press.

Véronique, D. (2005). Les interrelations entre la recherche sur l'acquisition de français langue étrangère et la didactique du français langue étrangère: Quelques pistes de travail. Acquisition et interaction en langue étrangère, 23, 9-41. 
Welcomme, A. (2013). La jonction interpropositionnelle à l'oral et à l'écrit en français langue étrangère. Thèse de doctorat. Bruxelles : Vrije Universiteit Brussel. 